\title{
Syndromic Surveillance for Situation Awareness: Understanding Syndrome Performance
}

\author{
Kristin Arkin ${ }^{\star 1,2}$ \\ ${ }^{1}$ Centers for Disease Control and Prevention, Atlanta, GA, USA; ${ }^{2}$ daho Division of Public Health, Boise, ID, USA
}

\section{Objective}

In August 2017, a large influx of visitors was expected to view the total solar eclipse in Idaho. The Idaho Syndromic Surveillance program planned to enhance situation awareness during the event. In preparation, we sought to examine syndrome performance of several newly developed chief complaint and combination chief complaint and diagnosis code syndrome definitions to aid in interpretation of syndromic surveillance data during the event.

\section{Introduction}

The August 21, 2017 total solar eclipse in Idaho was anticipated to lead to a large influx of visitors in many communities, prompting a widespread effort to assure Idaho was prepared. To support these efforts, the Idaho Syndromic Surveillance program (ISSp) developed a plan to enhance situation awareness during the event by conducting syndromic surveillance using emergency department (ED) visit data contributed to the National Syndromic Surveillance Program's BioSense platform by Idaho hospitals. ISSp sought input on anticipated threats from state and local emergency management and public health partners, and selected 8 syndromes for surveillance.

Ideally, the first electronic message containing information on an emergency department visit is sent to ISSp within 24 hours of the visit and includes the chief complaint for the visit. Data on other variables, such as diagnosis codes, are updated by subsequent messages for several days after the visit. Chief complaint (CC) text and discharge diagnosis (DD) codes are the primary variables used for syndrome match; delay in reporting these variables adversely affects timely syndrome match of visits. Because our plan included development of new syndrome definitions and querying data within 24 hours of visits, earlier than ISSp had done previously for trend analysis, we sought to better understand syndrome performance.

\section{Methods}

We defined messages with completed CC and DD as the last message regarding a visit where term count increased from previous messages regarding that visit, indicating new information was added to the field. We retrospectively assessed the total number of ED visits and calculated the daily frequency of completed CC and DD by days since visit date for visits during June 1-July 31, 2017. Additionally, we calculated facility mean word count in CC fields by averaging the word count of parsed, complete CC fields for visits occurring June 1-July 31, 2017 for each facility.

During July 10-24, 2017, we calculated the daily frequency of visits occurring in the previous 90 days for total ED visits and syndrome-matched visits for 8 selected syndromes (heat-related illness; cold exposure; influenza-like-illness; nausea, vomiting, and diarrhea; animal/bug bites and stings; drowning/submersion; alcohol/ drug intoxication; and medication replacement). Syndrome-matched visits were defined as visits with $\mathrm{CC}$ or DD that match the syndrome definition. We calculated the percent of syndrome-matched visits by syndromes defined with CC or CC and DD combined (CCDD) over time. Syndromes with fewer than 5 matched visits were excluded from analysis.

\section{Results}

Complete CCs were received for $99.1 \%$ of visits and complete DDs were received for $89.8 \%$ of visits. Complete CCs were submitted for
$58.2 \%$ of visits within 1 day of the visit, $88.9 \%$ of visits within 3 days, and $98.9 \%$ of visits within 7 days. In contrast, complete DDs were submitted for $24.3 \%$ of visits within 1 day, $38.7 \%$ of visits within 3 days, and $53.7 \%$ of visits within 7 days (Table 1 ).

During the observation period, data submission from facilities representing approximately $33 \%$ of visits was interrupted for 5 $(36 \%)$ of 14 days. Heat-related illness, cold exposure, and drowning/ submersion, were excluded from syndrome-match analysis. During the 9 days of uninterrupted data submission, $100 \%$ syndrome-matched visits for syndromes defined by CC alone and $69.1 \%$ syndromematched visits for syndromes defined by CCDD were identified within 6-7 days of initial visit. Facilities with interrupted data submission contributed $75 \%$ of CC syndrome-matched visits and 33\% of CCDD syndrome-matched visits. The facility mean word count in CC fields from these facilities was $>15$ compared with 2-4 from other facilities.

\section{Conclusions}

Examination of syndrome performance prior to a known event quantitated differences in timeliness of CC and DD completeness and syndrome match. CCs and DDs in visit messages were not complete within 24 hours of initial visit. CC completion was nearly 34 percentage points greater than DD completeness 1 day after initial visit and did not converge until $\geq 15$ days after initial visit. Higher percentages of syndrome match within 6-7 days of initial visit were seen by $\mathrm{CC}$ alone than CCDD defined syndromes. Facilities using longer $\mathrm{CCs}$ contributed disproportionately to syndrome matching using CC, but not CCDD syndrome definitions. Syndromic surveillance system characteristics, including timeliness of CCs and DDs, length of $\mathrm{CCs}$, and characteristics of facilities from which data transmission is interrupted should be considered when building syndrome definitions that will be used for surveillance within 7 days of emergency department visits and when interpreting syndromic surveillance findings.

\begin{tabular}{|r|r|r|}
\hline & $\begin{array}{c}\text { Chief Complaint } \\
\mathrm{n}(\%)\end{array}$ & $\begin{array}{c}\text { Diagnosis Code } \\
\mathrm{n}(\%)\end{array}$ \\
\hline Complete fields & $\mathbf{2 7 , 6 2 8 ( 9 9 . 1 )}$ & $\mathbf{3 4 , 0 8 9}(\mathbf{8 9 . 8 )}$ \\
\hline$\leq 1$ day & $22,089(58.2)$ & $9,225(24.3)$ \\
\hline $2-3$ days & $11,667(30.7)$ & $5,464(14.4)$ \\
\hline $4-5$ days & $2,687(7.1)$ & $3,816(10.1)$ \\
\hline $6-7$ days & $1,055(2.8)$ & $1,876(4.9)$ \\
\hline $8-9$ days & $48(0.1)$ & $4,806(12.7)$ \\
\hline $10-14$ days & $44(0.1)$ & $8,223(21.7)$ \\
\hline$\geq 15$ days & $38(0.1)$ & $679(1.8)$ \\
\hline NULL & $342(0.9)$ & $3,881(10.2)$ \\
\hline Total & 37,970 & 37,970 \\
\hline
\end{tabular}

Table 1. Frequency of complete chief complaint (CC) and diagnosis code (DD) by number of days since visit date.

\section{Keywords}

Event surveillance; syndromic surveillance; situational awareness; situation awareness

\section{${ }^{*}$ Kristin Arkin}

E-mail: Kristinaarkin@gmail.com 